\title{
COHOMOLOGY THEOREMS FOR ASYMPTOTIC SHEAVES
}

\author{
JORGE MOZO FERNÁNDEZ
}

(Received December 8, 1997, revised November 18, 1998)

\begin{abstract}
In this paper, we study the sheaves $\mathcal{A}_{E}^{<0}$ and $\mathcal{A}_{E}^{<-\kappa}$ of strongly asymptotically developable functions with null expansion, which are subsheaves of $\mathcal{A}$ defined by Majima. Following the method developed in one variable by Sibuya, and in several variables by Majima, we compute the first cohomology group of the $n$-torus and the boundary of the real blow-up with coefficients in these sheaves. The same technique is used to study the multiplicative case (sheaves of non-abelian groups), in order to calculate the first cohomology set. This generalizes previous results of Majima, Haraoka and Zurro.
\end{abstract}

1. Definitions and notations. A polysector $V=V_{1} \times \cdots \times V_{n}$ in $\boldsymbol{C}^{n}$ is a product of open sectors, an open sector being a set of the type

$$
V_{\alpha, \beta, R}=\{z \in C|\alpha<\arg z<\beta, 0<| z \mid<R\},
$$

where $R \in(0, \infty)$. The number $\beta-\alpha$ is the opening of $V_{\alpha, \beta, R}$. A subpolysector $W$ of $V$ $(W<V)$ is $W_{1} \times \cdots \times W_{n}$, where $W_{i}$ is a closed sector of finite radius and strictly smaller opening than $V_{i}$.

$\mathcal{A}(V)$ will denote the $C$-algebra of functions that are strongly asymptotically developable in $V$, introduced by Majima in [M1]. Let us recall that $f \in \mathcal{A}(V)$ if and only if there exist a family of functions

$$
\mathcal{F}=\left\{f_{\alpha_{J}}\left(z_{J^{c}}\right) \in \mathcal{O}\left(V_{J^{c}}\right) \mid \emptyset \neq J \subseteq\{1, \ldots, n\}, \alpha_{J} \in N^{J}\right\}
$$

such that, if $W<V$ and $N \in N^{n}$, there exists $C_{W, N}>0$ with

$$
\left|f(z)-\operatorname{App}_{N}(\mathcal{F})(z)\right|<C_{W, N} \cdot\left|z^{N}\right| \text { in } W,
$$

where

$$
\operatorname{App}_{N}(\mathcal{F})(z)=\sum_{\emptyset \neq J \subseteq\{1, \ldots, n\}} \sum_{j \in J} \sum_{\alpha_{J}<N_{J}}(-1)^{\sharp J+1} \cdot f_{\alpha_{J}}\left(z_{J^{c}}\right) \cdot z_{J}^{\alpha_{J}}
$$

and $J^{c}=\{1, \ldots, n\} \backslash J$. We have used the following notations: if $J \in\{1, \ldots, n\}, V_{J}:=$ $\prod_{j \in J} V_{j}$, and $z_{J}$ is the element of $V_{J}$ obtained by projection of $z \in V$ to $V_{J}$. This family $\mathcal{F}$ (the total family of coefficients of $f$ ) is unique, and it will be denoted by $T A(f)$. As in [M1], for $f \in \mathcal{A}(V), \emptyset \neq J \subseteq\{1, \ldots, n\}, F A_{J}(f)$ will denote the series

$$
F A_{J}(f)=\sum_{\alpha_{J} \in N^{J}} f_{\alpha_{J}}\left(z_{J^{c}}\right) z_{J}^{\alpha_{J}} \in \mathcal{A}\left(V_{J^{c}}\right)\left[\left[z_{J}\right]\right]
$$

1991 Mathematics Subject Classification. Primary 41A60; Secondary 55N30.

Partially supported by the TMR (Training and Mobility Researchers) European Network ERBFMRXCT960040 and the DGICYT (Dirección General de Investigación Científica y Técnica).

The author wants to thank the referee and the editorial office for their remarks. 
If $J=\{1, \ldots, n\}$, we shall write $F A(f)$ instead of $F A_{J}(f)$.

For the main properties of $\mathcal{A}(V)$ the reader can see [M1, M2, Mo].

We shall use freely multi-index notations, such us $z^{\alpha}:=z_{1}^{\alpha_{1}} \cdots z_{n}^{\alpha_{n}}, \ldots$, as we have already done. Also, if $\alpha, \beta \in N^{n}, \alpha<\beta$ will mean that each component of $\alpha$ is strictly smaller than the corresponding component of $\beta$.

If $s=\left(s_{1}, \ldots, s_{n}\right) \in \boldsymbol{R}_{\geq 0}^{n}, \mathcal{A}_{s}(V)$ is the $C$-subalgebra of $\mathcal{A}(V)$ of $s$-Gevrey type functions (see [Ha]). This means that, with previous notations, one can choose $C_{W, N}=$ $C_{W}^{\prime} \cdot A_{W}^{N} \cdot N !^{s}$, for certain $C_{W}^{\prime}>0$ and $A_{W} \in\left(\boldsymbol{R}_{>0}\right)^{n}$, independent of $N$.

As usual, $\mathcal{O}$ will be the ring of germs of holomorphic functions at the origin, and $\mathcal{O}(U)$ the set of holomorphic functions defined in the open set $U$. This defines a sheaf, that we shall also denote by $\mathcal{O}$.

Fixing coordinates $z_{1}, \ldots, z_{n}, E$ will be a (germ of) normal crossing divisor or a linear subvariety. So, if $H_{i}$ is the hyperplane defined by the equation $z_{i}=0$ for $i=1, \ldots, k$, then $E=H_{1} \cup \cdots \cup H_{k}$ or $E=H_{1} \cap \cdots \cap H_{k} . \mathcal{A}_{E}^{<0}(V)$ is the subset of $\mathcal{A}(V)$ whose elements vanish on $E$, i.e., equivalently,

1. If $f \in \mathcal{A}(V)$ and $T A(f)=\left\{f_{\alpha_{J}}\left(z_{J^{c}}\right) \in \mathcal{A}\left(V_{J^{c}}\right) \mid \alpha_{J} \in N^{J}\right\}$ is the total family of coefficients of asymptotic development for $f$, then

(a) if $E=H_{1} \cup \ldots \cup H_{k}, f_{\alpha_{J}}\left(z_{J^{c}}\right)=0$ whenever $\{1, \ldots, k\} \cap J \neq \emptyset$,

(b) if $E=H_{1} \cap \cdots \cap H_{k}, f_{\alpha_{J}}\left(z_{J^{c}}\right)=0$ whenever $\{1, \ldots, k\} \subseteq J$.

2. If $W<V$, there exists a $C^{\infty}$ extension $F$ of $\left.f\right|_{W}$ such that $F \equiv 0$ on $E$.

This equivalence is an easy consequence of the results in [Z].

Obviously, $E_{1} \subseteq E_{2} \Leftrightarrow \mathcal{A}_{E_{2}}^{<0}(V) \subseteq \mathcal{A}_{E_{1}}^{<0}(V)$. We define analogously, if $\kappa=1 / s=$ $\left(1 / s_{1}, \ldots, 1 / s_{n}\right), \mathcal{A}_{E}^{\leq-\kappa}(V)=\mathcal{A}_{E}^{<0}(V) \cap \mathcal{A}_{s}(V)$ (we keep the usual notations of the 1 dimensional case used by Malgrange and Ramis).

Let $\pi: \tilde{C} \rightarrow C$ be the real blow-up of $\boldsymbol{C}$ at 0 , with $\pi^{-1}(0)=S^{1}$, the points of $S^{1}$ representing real directions from 0 in $\boldsymbol{C}$. $\tilde{\boldsymbol{C}}^{n}$ will be the componentwise real blow-up (we blow up each component separately, see [M2, p. 37]). It is a real manifold with boundary $\partial \tilde{\boldsymbol{C}}^{n}$, where $\partial \tilde{\boldsymbol{C}}^{n}$ is the inverse image by $\pi_{n}: \tilde{\boldsymbol{C}}^{n} \rightarrow \boldsymbol{C}^{n}$ of the coordinate hyperplanes $H_{1} \cap \cdots \cap H_{n}$. Let us put $X_{i}=\pi_{n}^{-1}\left(H_{i}\right)$. So, $\partial \tilde{\boldsymbol{C}}^{n}=X_{1} \cup \cdots \cup X_{n}$. Over $\tilde{\boldsymbol{C}}^{n}$ we define sheaves $\mathcal{A}, \mathcal{A}_{s}, \mathcal{A}_{E}^{<0}, \mathcal{A}_{E}^{\leq-\kappa}$ from the above definitions. If $z \notin \partial \tilde{\boldsymbol{C}}^{n}$, the stalk $\mathcal{F}_{z}(\mathcal{F}$ being one of the above sheaves) coincides with $\mathcal{O}$. So we are mainly interested in the study of these sheaves over $\partial \tilde{\boldsymbol{C}}^{n}$. In particular, we shall compute the first cohomology group of $\mathcal{A}_{E}^{<0}, \mathcal{A}_{E}^{\leq-\kappa}$ following the method developed by Sibuya. We shall need to compute $H^{1}$ also over $\boldsymbol{T}^{n}=X_{1} \cap \cdots \cap X_{n}$. In this case, some of the results had already been obtained by Majima [M1], Haraoka [Ha] and Zurro [Z] (the last author uses a different method, following Malgrange in [Ml]). We will reprove them in order to obtain a global vision.

Given a Fréchet space $L, \mathcal{A}(V ; L), \mathcal{A}_{s}(V ; L), \ldots$ will denote the sets of strongly asymptotically developable functions with values in $L$ (so, $\mathcal{A}(V)=\mathcal{A}(V ; C)$ ), with obvious modifications of the definitions. The set $\mathcal{A}(V)$ is itself a Fréchet space, where a family of seminorms is given by

$$
p_{W, N}(f)=\sup \left\{\left|D^{N} f(z)\right| / z \in W\right\},
$$


and in fact we have canonical isomorphisms $\mathcal{A}\left(V_{1} ; \mathcal{A}\left(V_{2}\right)\right) \cong \mathcal{A}\left(V_{1} \times V_{2}\right)$. Precise details of this approach can be read in [He]. $L\{z\}$ will denote the $C$-algebra of convergent series with coefficients in $L$, i.e., if $\sum a_{\alpha} z^{\alpha} \in L[[z]]$ and $p$ is a continuous seminorm on $L, \sum p\left(a_{\alpha}\right) z^{\alpha} \in$ $C\{z\}$.

\section{First cohomology group of the asymptotic sheaves.}

2.1. Main lemma. The following result generalizes Cartan's decomposition lemma of complex analysis.

LEMMA 2.1. Let $f \in \mathcal{A}_{\left(z_{1}=0\right)}^{<0}$. If $V_{1}=\left\{z_{1} \in C\left|a<\arg z_{1}<b,\right| z_{1} \mid<R\right\}$, denote $\tilde{V}_{1}=\{z \in C|a<\arg z<b+2 \pi| z \mid,<R\}$. Then there exist $F \in \mathcal{A}\left(\tilde{V}_{1} \times V_{2} \times \cdots \times V_{n}\right)$ such that

$$
F\left(z_{1} e^{2 \pi i}, z_{2}, \ldots, z_{n}\right)-F\left(z_{1}, \ldots, z_{n}\right)=f(z)
$$

If $1 \notin J$, then

$$
F_{\alpha_{J}}\left(z_{1} e^{2 \pi i}, z_{J^{c} \backslash\{1\}}\right)-F_{\alpha_{J}}\left(z_{J^{c}}\right)=f_{\alpha_{J}}\left(z_{J^{c}}\right) .
$$

If in addition, $f \in \mathcal{A}_{s}(V), F \in \mathcal{A}_{s}\left(\tilde{V}_{1} \times V_{2} \times \cdots \times V_{n}\right)$.

SKETCH OF PROOF. As in the case of one variable (see [S]), we define $F(z)$ as the Cauchy-Heine transform (with parameters) of $f$ in the first variable. This $F$ verifies the required properties.

2.2. Cohomology over $T^{n}$. A good covering of $S^{1}$ will be a covering by (at least three) open intervals, each three of them having empty intersection. Every interval in $S^{1}$ represents a sector at the origin in $\boldsymbol{C}$, forgetting the radius. A good covering of $\boldsymbol{T}^{n}$ is a product of good open coverings of $S^{1}$. As every open covering of $T^{n}$ can be refined by a good covering $\mathcal{U}$, we only need to compute $H^{1}(\mathcal{U} ; *)$. In the sequel, we shall identify sectors with intervals of $S^{1}$.

Proposition 2.2. Let $\mathcal{V}=\left\{V_{1}, \ldots, V_{r}\right\}$ be a good covering of $S^{1}$, and $V^{\prime}$ a polysector in $C^{n-1}$. If $V_{i j}=V_{i} \cap V_{j}$, let $f_{i, i+1} \in \mathcal{A}\left(V_{i, i+1} \times V^{\prime}\right)$ such that

$$
\sum_{i} F A_{\{1\}}\left(f_{i, i+1}\right)=0 \in \mathcal{A}\left(V^{\prime}\right)\left[\left[z_{1}\right]\right] .
$$

Then, there exist $f_{i} \in \mathcal{A}\left(V_{i} \times V^{\prime}\right)$ such that $f_{i, i+1}=\left.f_{i+1}\right|_{V_{i, i+1} \times V^{\prime}}-\left.f_{i}\right|_{V_{i, i+1} \times V^{\prime}}$.

If $f_{i, i+1} \in \mathcal{A}_{s}\left(V_{i, i+1} \times V^{\prime}\right)$, then $f_{i} \in \mathcal{A}_{s}\left(V_{i} \times V^{\prime}\right)$ (provided that the openings of $V_{i}$ and $V^{\prime}$ are sufficiently small).

REMARK 2.3. One can observe that this is a reformulation of [M2, Th. 3.2. (iii)].

Proof OF PROPOSITION 2.2. By a theorem of Borel-Ritt, take $g_{i} \in \mathcal{A}\left(V_{i} \times V^{\prime}\right)$ such that

$$
F A_{\{1\}}\left(g_{i}\right)=\sum_{k=1}^{i-1} F A_{\{1\}}\left(f_{k, k+1}\right)
$$


$(i=2, \ldots, r+1=1)$. If $h_{i, i+1}=f_{i, i+1}-g_{i+1}+g_{i}, F A_{\{1\}}\left(h_{i, i+1}\right)=0$. Let us suppose first that $h_{i, i+1}=0$ if $i \neq 1$. Then, Lemma 2.1 gives us a function $H_{12}$, and we define $h_{i}=\left.H_{12}\right|_{V_{i} \times V^{\prime}}$. It follows that $h_{i+1}-h_{i}=h_{i, i+1}$.

In general, let $h_{j}^{i, i+1} \in \mathcal{A}\left(V_{j} \times V^{\prime}\right)$ such that

$$
h_{j+1}^{i, i+1}-h_{j}^{i, i+1}=\delta_{i j} h_{i, i+1} .
$$

The functions

$$
f_{i}=g_{i}+\sum_{i=1}^{r} h_{j}^{i, i+1}
$$

give the result. The Gevrey case is similar.

Proposition 2.2 is essentially a result in one variable, but it can be used in order to prove the corresponding assertion in several variables:

Proposition 2.4. Let $\mathcal{V}=\left\{V_{i_{1}} \times \cdots \times V_{i_{n}}\right\}$ be a good covering of $\boldsymbol{T}^{n}$. Then, the map

$$
H^{1}(\mathcal{V} ; \mathcal{A}) \rightarrow H^{1}\left(\mathcal{V} ; \mathcal{A} / \mathcal{A}_{E}^{<0}\right)
$$

is injective. Namely, for each $i=\left(i_{1}, \ldots, i_{n}\right), i^{\prime}=\left(i_{1}^{\prime}, \ldots, i_{n}^{\prime}\right)$, let $f_{i, i^{\prime}} \in \mathcal{A}\left(V_{i} \cap V_{i^{\prime}}\right)$ satisfying the cocycle condition, such that

$$
\left[\left(f_{i, i^{\prime}}\right)_{i, i^{\prime}}\right]=0 \text { as an element of } H^{1}\left(\mathcal{V} ; \mathcal{A} / \mathcal{A}_{E}^{<0}\right),
$$

$[\cdots]$ meaning the equivalence class. Then $\left[\left(f_{i, i^{\prime}}\right)_{i, i^{\prime}}\right]=0$ as an element of $H^{1}(\mathcal{V} ; \mathcal{A})$.

If $f_{i, i^{\prime}}(z) \in \mathcal{A}_{s}\left(V_{i} \cap V_{i^{\prime}}\right)$ and $\left[\left(f_{i, i^{\prime}}\right)_{i, i^{\prime}}\right]=0$ in $H^{1}\left(\mathcal{V} ; \mathcal{A}_{s} / \mathcal{A}_{E}^{\leq-\kappa}\right)$, then $\left[\left(f_{i, i^{\prime}}\right)_{i, i^{\prime}}\right]=0$ in $H^{1}\left(\mathcal{V} ; \mathcal{A}_{s}\right)$.

Proof. Suppose first that $E=(0)$. The exactness of the sequence

$$
0 \rightarrow \mathcal{A}_{E}^{<0} \rightarrow \mathcal{A} \rightarrow C[[z]] \rightarrow 0
$$

shows that $\mathcal{A} / \mathcal{A}_{E}^{<0} \cong \boldsymbol{C}[[z]]$ (Taylor map).

If $\tilde{\imath}$ and $\tilde{l}^{\prime}$ are $(n-1)$-tuples, we denote

$$
\begin{aligned}
& A_{\tilde{\imath}, \tilde{l}^{\prime}}=\sum_{k} F A_{\{1\}}\left(f_{(k, \tilde{l}),\left(k+1, \tilde{\imath}^{\prime}\right)}\right) \in \mathcal{A}\left(V_{\tilde{\imath}} \cap V_{\tilde{l}^{\prime}}\right)\left[\left[z_{1}\right]\right], \\
& B_{\tilde{\imath}, \tilde{i}^{\prime}}=\sum_{k} F A_{\{1\}}\left(f_{(k, \tilde{\imath}),\left(k, \tilde{i}^{\prime}\right)}\right) \in \mathcal{A}\left(V_{\tilde{\imath}} \cap V_{\tilde{l}^{\prime}}\right)\left[\left[z_{1}\right]\right] .
\end{aligned}
$$

The cocycle condition implies that

$$
f_{(k, \tilde{\imath}),\left(k, \tilde{i}^{\prime}\right)}+f_{\left(k, \tilde{i}^{\prime}\right),\left(k+1, \tilde{i}^{\prime}\right)}=f_{(k, \tilde{i}),(k+1, \tilde{\imath})}+f_{(k+1, \tilde{i}),\left(k+1, \tilde{i}^{\prime}\right)}
$$

and so

$$
B_{\tilde{\imath}, \tilde{l}^{\prime}}+A_{\tilde{l}^{\prime}, \tilde{l}^{\prime}}=A_{\tilde{i}, \tilde{l}}+B_{\tilde{i}, \tilde{l}^{\prime}} .
$$

In the intersection $V_{i} \cap V_{i^{\prime}}, A_{\tilde{\imath}, \tilde{l}}=A_{\tilde{l}^{\prime}, \tilde{l}^{\prime}}$ and so, they glue together in

$$
A \in \mathcal{O}\left(D_{2} \times \cdots \times D_{n}\right)\left[\left[z_{1}\right]\right] .
$$


The hypothesis shows that the Taylor series of $A$ at the origin is 0 , so $A=0=A_{\tilde{i}, \tilde{\imath}}$. Now we use a kind of argument that has been developed in [M1]. By the previous proposition, we can find $f_{\left(i_{1}, \tilde{\imath}\right)} \in \mathcal{A}\left(V_{i_{1}} \times V_{\tilde{\imath}}\right)$ such that

$$
f_{\left(i_{1}, \tilde{\imath}\right),\left(i_{1}^{\prime}, \tilde{i}\right)}=f_{\left(i_{1}^{\prime}, \tilde{i}\right)}-f_{\left(i_{1}, \tilde{\imath}\right)}
$$

and

$$
\sum_{i_{1}} f_{\left(i_{1}, \tilde{i}\right), \alpha_{J}}\left(z_{J^{c}}\right)=0 \quad \text { if } 1 \notin J
$$

Again by the cocycle condition, the equality

$$
f_{\left(i_{1}, \tilde{i}\right),\left(i_{1}^{\prime}, \tilde{i}^{\prime}\right)}-f_{\left(i_{1}^{\prime}, \tilde{i}^{\prime}\right)}+f_{\left(i_{1}, \tilde{i}\right)}=f_{\left(i_{1}^{\prime \prime}, \tilde{i}\right),\left(i_{1}^{\prime \prime \prime}, \tilde{i}^{\prime}\right)}-f_{\left(i_{1}^{\prime \prime \prime}, \tilde{i}^{\prime}\right)}+f_{\left(i_{1}^{\prime \prime}, \tilde{i}\right)}
$$

holds in the intersection of the domains (for indices $i_{1}, i_{1}^{\prime}, i_{1}^{\prime \prime}, i_{1}^{\prime \prime \prime}$ and $(n-1)$-tuples $\tilde{i}, \tilde{\imath}^{\prime}$ ). For, add $f_{\left(i_{1}, \tilde{i}\right),\left(i_{1}^{\prime}, \tilde{i}^{\prime}\right)}+f_{\left(i_{1}^{\prime \prime}, \tilde{\imath}\right)}=f_{\left(i_{1}, \tilde{i}\right),\left(i_{1}^{\prime}, \tilde{i}^{\prime}\right)}+f_{\left(i_{1}, \tilde{i}\right),\left(i_{1}^{\prime \prime}, \tilde{i}\right)}+f_{\left(i_{1}, \tilde{i}\right)}$ to each side of the equality $f_{\left(i_{1}^{\prime}, \tilde{i}^{\prime}\right)}+f_{\left(i_{1}^{\prime}, \tilde{i}^{\prime}\right),\left(i_{1}^{\prime \prime \prime}, \tilde{i}^{\prime}\right)}=f_{\left(i_{1}^{\prime \prime \prime}, \tilde{i}^{\prime}\right)}$. By glueing together the preceding functions, we obtain $f_{\left(\tilde{\imath}, \tilde{l}^{\prime}\right)} \in \mathcal{A}\left(D_{1} \times\left(V_{\tilde{\imath}} \cap V_{\tilde{\imath}^{\prime}}\right)\right)$.

We keep the same conditions as in the beginning of the proof (identify $\mathcal{A}(D \times V) \cong$ $\mathcal{A}(V ; \mathcal{O}(D))$ and consider asymptotic developments with values in the Fréchet space $\mathcal{O}(D))$. So, for each $(n-2)$-tuple $\bar{l}$, construct functions

$$
f_{\left(i_{2}, \bar{i}\right)} \in \mathcal{A}\left(D_{1} \times V_{i_{2}} \times V_{\bar{l}}\right)
$$

such that

$$
f_{\left(i_{2}^{\prime}, \bar{l}\right)}-f_{\left(i_{2}, \bar{l}\right)}=f_{\left(i_{2}, \bar{l}\right),\left(i_{2}^{\prime}, \bar{l}\right)}
$$

and we iterate the process, obtaining for $(n-k)$-tuples $j=\left(j_{k+1}, \ldots, j_{n}\right), j^{\prime}=\left(j_{k+1}^{\prime}, \ldots, j_{n}^{\prime}\right)$, and indices $j_{k}, j_{k}^{\prime}$, functions

$$
\begin{gathered}
f_{j} \in \mathcal{A}\left(D_{1} \times \cdots \times D_{k} \times V_{j_{k+1}} \times \cdots \times V_{j_{n}}\right), \\
f_{\left(j_{k}, j\right),\left(j_{k}^{\prime}, j^{\prime}\right)} \in \mathcal{A}\left(D_{1} \times \cdots \times D_{k-1} \times\left(V_{j_{k}} \cap V_{j_{k}^{\prime}}\right) \times\left(V_{j} \cap V_{j^{\prime}}\right)\right)
\end{gathered}
$$

such that

$$
f_{j, j^{\prime}}=f_{\left(j_{k}, j\right),\left(j_{k}^{\prime}, j^{\prime}\right)}-f_{\left(j_{k}, j^{\prime}\right)}+f_{\left(j_{k}, j\right)}
$$

Defining

$$
F_{\left(j_{1}, \ldots, j_{n}\right)}=f_{\left(j_{1}, \ldots, j_{n}\right)}+f_{\left(j_{2}, \ldots, j_{n}\right)}+\cdots+f_{j_{n}},
$$

a straightforward computation shows the required property, i.e.,

$$
F_{j^{\prime}}-F_{j}=f_{j, j^{\prime}} .
$$

Consider now a general $E$. We have an exact diagram

$$
\begin{array}{ccccccccc}
0 & \rightarrow & \mathcal{A}_{E}^{<0} & \rightarrow & \mathcal{A} & \rightarrow & \mathcal{A} / \mathcal{A}_{E}^{<0} & \rightarrow & 0 \\
& & \downarrow & & \downarrow & & \downarrow & & \\
0 & & \mathcal{A}_{(0)}^{<0} & \rightarrow & \mathcal{A} & \rightarrow & C[[z]] & \rightarrow & 0,
\end{array}
$$


from which we deduce an exact cohomology sequence (taking global sections)

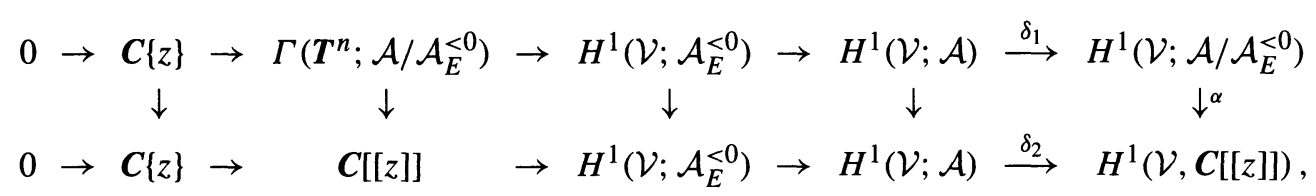

We have shown that $\delta_{2}=\alpha \circ \delta_{1}$ is injective, so $\delta_{1}$ is also injective. The same argument applies to the Gevrey case.

REMARK 2.5. The spaces $\Gamma\left(\boldsymbol{T}^{n} ; \mathcal{A} / \mathcal{A}_{E}^{<0}\right)$ may be computed explicitly. Moreover, if we write

$$
\hat{f}(z)=\sum_{\alpha} \hat{f}_{\alpha}\left(z_{1}, \ldots, \hat{z}_{i}, \ldots, z_{n}\right) \cdot z_{i}^{\alpha}
$$

and $E=H_{1} \cup \cdots \cup H_{k}$, it can be canonically identified with the set of series $\hat{f} \in C[[z]]$ such that if $1 \leq i \leq k$, then there exists a disk $D$ around the origin in $C^{n-1}$ such that $\hat{f}_{\alpha} \in \mathcal{O}(D)$. This set is precisely the stalk at the origin of the formal completion of the sheaf $\mathcal{O}$ along the divisor $E$ (see $[\mathrm{Gr}])$, i.e.,

$$
\left(\overleftarrow{\lim }_{k} \mathcal{O} / \mathcal{I}_{E}^{k}\right)_{0} .
$$

If $E$ is a linear subvariety $H_{1} \cap \cdots \cap H_{k}$, the above stalk is precisely

$$
\underset{D \rightarrow 0}{\lim _{D \rightarrow 0}} \mathcal{O}\left(D_{k+1} \times \cdots \times D_{n}\right)\left[\left[z_{1}, \ldots, z_{k}\right]\right]
$$

i.e., the set of formal power series

$$
\sum f_{\alpha_{1}, \ldots, \alpha_{k}}\left(z_{k+1}, \ldots, z_{n}\right) \cdot z_{1}^{\alpha_{1}} \cdots z_{k}^{\alpha_{k}}
$$

such that $f_{\alpha}\left(z_{k+1}, \ldots, z_{n}\right) \in \mathcal{O}(D), D$ being a disk in $C^{n-k}$.

Analogously, $\Gamma\left(\boldsymbol{T}^{n} ; \mathcal{A}_{s} / \mathcal{A}_{E}^{\leq-\kappa}\right)$ is identified with the "formal Gevrey completion", i.e., if $E$ is a normal crossing divisor $\left(z_{1} \cdots z_{k}=0\right)$, the set of series $(*)$ such that, if $K$ is a compact set in $D$,

$$
\sum\left\|\hat{f}_{\alpha}\left(z_{1}, \ldots, \hat{z}_{i}, \ldots, z_{n}\right)\right\|_{K} \cdot z_{i}^{\alpha} \in C\left[\left[z_{i}\right]\right]_{s_{i}}
$$

and when $E$ is the linear subvariety $H_{1} \cap \cdots \cap H_{k}$ we need in (**) that

$$
\sum\left\|f_{\alpha_{1}, \ldots, \alpha_{k}}\left(z_{k+1}, \ldots, z_{n}\right)\right\|_{K} \cdot z_{1}^{\alpha_{1}} \cdots z_{n}^{\alpha_{n}} \in C\left[\left[z_{1}, \ldots, z_{k}\right]\right]_{\left(s_{1}, \ldots, s_{k}\right)}
$$

for every compact set $K$ in $D$.

THEOREM 2.6. There are canonical isomorphisms

$$
\begin{gathered}
H^{1}\left(\boldsymbol{T}^{n} ; \mathcal{A}_{E}^{<0}\right) \cong \Gamma\left(\boldsymbol{T}^{n} ; \mathcal{A} / \mathcal{A}_{E}^{<0}\right) / \boldsymbol{C}\{z\}, \\
H^{1}\left(\boldsymbol{T}^{n} ; \mathcal{A}_{E}^{\leq-\kappa}\right) \cong \Gamma\left(\boldsymbol{T}^{n} ; \mathcal{A}_{s} / \mathcal{A}_{E}^{\leq-\kappa}\right) / \boldsymbol{C}\{z\} .
\end{gathered}
$$

ProOF. Take a good covering $\mathcal{V}$ of $\boldsymbol{T}^{n}$, and the long exact sequences

$$
0 \rightarrow \boldsymbol{C}\{z\} \rightarrow \Gamma\left(\boldsymbol{T}^{n} ; \mathcal{A} / \mathcal{A}_{E}^{<0}\right) \rightarrow H^{1}\left(\boldsymbol{T}^{n} ; \mathcal{A}_{E}^{<0}\right) \rightarrow H^{1}\left(\boldsymbol{T}^{n} ; \mathcal{A}\right) \stackrel{\delta}{\longrightarrow} H^{1}\left(\boldsymbol{T}^{n} ; \mathcal{A} / \mathcal{A}_{E}^{<0}\right),
$$


$0 \rightarrow \boldsymbol{C}\{z\} \rightarrow \Gamma\left(\boldsymbol{T}^{n} ; \mathcal{A}_{s} / \mathcal{A}_{E}^{\leq-\kappa}\right) \rightarrow H^{1}\left(\boldsymbol{T}^{n} ; \mathcal{A}_{E}^{\leq-\kappa}\right) \rightarrow H^{1}\left(\boldsymbol{T}^{n} ; \mathcal{A}_{s}\right) \stackrel{\delta}{\longrightarrow} H^{1}\left(\boldsymbol{T}^{n} ; \mathcal{A}_{s} / \mathcal{A}_{E}^{\leq-\kappa}\right)$.

The assertion of the theorem is equivalent to the injectivity of $\delta$, that is assured by the previous proposition.

REMARK 2.7. When $E=H_{1} \cup \cdots \cup H_{n}$, this result can be seen in [M1] and [Ha]. When $E=H_{1} \cap \cdots \cap H_{n}$, it is proved in [Z] using a different method, only in the general case (not Gevrey).

2.3. Cohomology over $\partial \tilde{\boldsymbol{C}}^{n}$. In order to compute $H^{1}\left(\partial \tilde{\boldsymbol{C}}^{n} ; *\right)$, where $*$ is one of the sheaves $\mathcal{A}_{E}^{<0}, \mathcal{A}_{E}^{\leq-\kappa}$, we write $\partial \tilde{\boldsymbol{C}}^{n}=X_{1} \cup \cdots \cup X_{n}$ as in the beginning of the paper, and we shall apply a Mayer-Vietoris argument. At the end, we will remark the local case.

We need a previous technical lemma:

LEMMA 2.8. If $h \in \bigcap_{k=2}^{n} \mathcal{O}\left(\boldsymbol{C}^{n-2}\right)\left\{z_{1}, z_{k}\right\}$, there exist $h_{1} \in \bigcap_{k=2}^{n} \mathcal{O}\left(\boldsymbol{C}^{n-1}\right)\left\{z_{k}\right\}$, $h_{2} \in \mathcal{O}\left(C^{n-1}\right)\left\{z_{1}\right\}$ with $h_{1}-h_{2}=h$.

PROOF. $h$ may be considered as a holomorphic function defined in a neighbourhood $U$ of $\bigcup_{k=2}^{n} H_{1} \cap H_{k}$ in $C^{n}$. Let us suppose that $U$ is a logarithmically convex Reinhardt domain (this is always possible because they form a fundamental system of neighbourhoods of the origin). We can find open subsets $U_{1}$ and $U_{2}$ of $C^{n}$ such that $U_{1}$ is a neighbourhood of $\bigcup_{k=2}^{n} H_{k}$ and $U_{2}$ is a neighbourhood of $H_{1}$.

If $\mathcal{U}=\left\{U_{1}, U_{2}\right\}$ is an open covering of $U_{1} \cup U_{2}$, since

$$
H^{1}(\mathcal{U} ; \mathcal{O}) \hookrightarrow H^{1}\left(U_{1} \cup U_{2} ; \mathcal{O}\right)=0,
$$

we can find $h_{i} \in \mathcal{O}\left(U_{i}\right)$ such that $h_{1}-h_{2}=h$. These are the required functions.

THEOREM 2.9. There are isomorphisms

$$
\begin{gathered}
H^{1}\left(\partial \tilde{\boldsymbol{C}}^{n} ; \mathcal{A}_{E}^{<0}\right) \cong \Gamma\left(\partial \tilde{\boldsymbol{C}}^{n} ; \mathcal{A} / \mathcal{A}_{E}^{<0}\right) / \bigcap_{i=1}^{n} \mathcal{O}\left(\boldsymbol{C}^{n-1}\right)\left\{z_{i}\right\} \\
H^{1}\left(\partial \tilde{\boldsymbol{C}}^{n} ; \mathcal{A}_{E}^{\leq-\kappa}\right) \cong \Gamma\left(\partial \tilde{\boldsymbol{C}}^{n} ; \mathcal{A}_{s} / \mathcal{A}_{E}^{\leq-\kappa}\right) / \bigcap_{i=1}^{n} \mathcal{O}\left(\boldsymbol{C}^{n-1}\right)\left\{z_{i}\right\} .
\end{gathered}
$$

Proof. First of all, we will compute $H^{1}\left(X_{i} ; *\right)$ with $*$ one of the above sheaves. Suppose $i=1$. Recall that the real blow-up $\tilde{\boldsymbol{C}}^{n}$ of $\boldsymbol{C}^{n}$ is constructed as a product of $n$ times the real blow-up of $\boldsymbol{C}$. This latter is a product $S^{1} \times \boldsymbol{R}_{\geq 0}$ (polar coordinates). So, a good covering of $X_{1}$ is composed by open sets such as

$$
U=I_{i} \times\left(I_{j_{2}}^{2} \times A_{k_{2}}^{2}\right) \times \cdots \times\left(I_{j_{n}}^{n} \times A_{k_{n}}^{n}\right),
$$

where the I's are open intervals in $S^{1}$ and the $A$ 's in $\boldsymbol{R}_{\geq 0}$ (it is a product of good coverings of $S^{1}, \boldsymbol{R}_{\geq 0}$ ). We reorder the indices in such a way that $0 \in A_{k_{l}}^{l} \Leftrightarrow k_{l}=0$.

As before, we are going to prove that the natural maps

$$
\begin{gathered}
H^{1}\left(X_{1} ; \mathcal{A}\right) \stackrel{\delta}{\longrightarrow} H^{1}\left(X_{1} ; \mathcal{A} / \mathcal{A}_{E}^{<0}\right), \\
H^{1}\left(X_{1} ; \mathcal{A}_{s}\right) \stackrel{\delta}{\longrightarrow} H^{1}\left(X_{1} ; \mathcal{A}_{s} / \mathcal{A}_{E}^{\leq-\kappa}\right)
\end{gathered}
$$


are injective. Suppose $E=0$. In this case, if $z=\left(z_{1}, \ldots, z_{n}\right) \in X_{1}$ and

$$
J=\left\{j \in\{2, \ldots, n\} \mid z_{j} \in S^{1} \times\{0\} \subseteq \tilde{C}\right\},
$$

then the sheaves of coefficients of the right hand side are

$$
\begin{aligned}
\left(\mathcal{A} / \mathcal{A}_{E}^{<0}\right)_{z} & =\mathcal{O}_{\{2, \ldots, n\} \backslash J}\left[\left[z_{1}, z_{J}\right]\right], \\
\left(\mathcal{A}_{s} / \mathcal{A}_{E}^{\leq-\kappa}\right)_{z} & =\mathcal{O}_{\{2, \ldots, n\} \backslash J}\left[\left[z_{1}, z_{J}\right]\right]_{s} .
\end{aligned}
$$

Take

$$
\left[f_{i,(j, k)}^{i^{\prime},\left(j^{\prime}, k^{\prime}\right)} \in \Gamma\left(\left(I_{i} \cap I_{i^{\prime}}\right) \times\left(\prod_{l=2}^{n}\left(I_{j_{l}}^{l} \times A_{k_{l}}^{l}\right) \cap\left(I_{j_{l}^{\prime}}^{l} \times A_{k_{l}^{\prime}}^{l}\right)\right), *\right)\right] \in \operatorname{ker} \delta .
$$

Fixing $i$,

$$
\left[\left\{f_{i,(j, k)}^{i,\left(j^{\prime}, k^{\prime}\right)}\right\}_{(j, k),\left(j^{\prime}, k^{\prime}\right)}\right]
$$

can be seen as a 1-cocycle over $\tilde{\boldsymbol{C}}^{n-1}$, with parameters. We need here the following result, that we shall prove later. Remark that the first assertion is a particular case of [M2, Chapter 1, Theorem 3.3].

PROPOSITION 2.10.

$$
\begin{gathered}
H^{1}\left(\tilde{\boldsymbol{C}}^{n} ; \mathcal{A}_{E}^{<0}\right) \cong \Gamma\left(\partial \tilde{\boldsymbol{C}}^{n} ; \mathcal{A} / \mathcal{A}_{E}^{<0}\right) / \mathcal{O}\left(\boldsymbol{C}^{n}\right), \\
H^{1}\left(\tilde{\boldsymbol{C}}^{n} ; \mathcal{A}_{E}^{\leq-\kappa}\right) \cong \Gamma\left(\partial \tilde{\boldsymbol{C}}^{n} ; \mathcal{A}_{s} / \mathcal{A}_{E}^{\leq-\kappa}\right) / \mathcal{O}\left(\boldsymbol{C}^{n}\right) .
\end{gathered}
$$

Then, there are $f_{i,(j, k)}$ on $I \times \prod_{l=2}^{n}\left(I_{j_{l}} \times A_{k_{l}^{l}}\right)$ such that

$$
f_{i,(j, k)}^{i,\left(j^{\prime}, k^{\prime}\right)}=f_{i,\left(j^{\prime}, k^{\prime}\right)}-f_{i,(j, k)} .
$$

As in Proposition 2.4, the equality

$$
f_{i,(j, k)}^{i^{\prime},\left(j^{\prime}, k^{\prime}\right)}-f_{i^{\prime},\left(j^{\prime}, k^{\prime}\right)}+f_{i,(j, k)}=f_{i,\left(j^{\prime \prime}, k^{\prime \prime}\right)}^{i^{\prime},\left(j^{\prime \prime \prime}, k^{\prime \prime \prime}\right)}-f_{i^{\prime},\left(j^{\prime \prime \prime}, k^{\prime \prime \prime}\right)}+f_{i,\left(j^{\prime \prime}, k^{\prime \prime}\right)}
$$

defines $F_{i, i^{\prime}} \in \mathcal{A}_{E}^{<0}\left(I_{i} \cap I_{i^{\prime}} ; \mathcal{O}\left(\boldsymbol{C}^{n-1}\right)\right)$ and hence $F_{i, i^{\prime}}=F_{i^{\prime}}-F_{i}$ with $F_{i} \in \mathcal{A}\left(I_{i} ; \mathcal{O}\left(C^{n-1}\right)\right)$. The family of functions

$$
g_{i,(j, k)}:=f_{i,(j, k)}+F_{i}
$$

has as coboundary the desired cocycle. The Gevrey case is analogous, and so,

$$
\begin{aligned}
H^{1}\left(X_{1} ; \mathcal{A}_{E}^{<0}\right) & \cong C[[z]] / \mathcal{O}\left(C^{n-1}\right)\left\{z_{1}\right\}, \\
H^{1}\left(X_{1} ; \mathcal{A}_{E}^{\leq-\kappa}\right) & \cong C[[z]]_{s} / \mathcal{O}\left(C^{n-1}\right)\left\{z_{1}\right\} .
\end{aligned}
$$

Now we apply induction on $n$. Suppose that

$$
\begin{gathered}
H^{1}\left(X_{2} \cup \cdots \cup X_{n} ; \mathcal{A}_{E}^{<0}\right) \cong C[[z]] / \bigcap_{k=2}^{n} \mathcal{O}\left(\boldsymbol{C}^{n-1}\right)\left\{z_{k}\right\}, \\
H^{1}\left(X_{1} \cap\left(X_{2} \cup \cdots \cup X_{n}\right) ; \mathcal{A}_{E}^{<0}\right) \cong C[[z]] / \bigcap_{k=2}^{n} \mathcal{O}\left(\boldsymbol{C}^{n-2}\right)\left\{z_{1}, z_{k}\right\} .
\end{gathered}
$$


The exact Mayer-Vietoris sequence [I]

$$
\begin{aligned}
0 \rightarrow H^{1}\left(\partial \tilde{\boldsymbol{C}}^{n} ; \mathcal{A}_{E}^{<0}\right) \rightarrow H^{1}\left(X_{1} ; \mathcal{A}_{E}^{<0}\right) \oplus H^{1}\left(X_{2} \cup\right. & \left.\cdots \cup X_{n} ; \mathcal{A}_{E}^{<0}\right) \\
& \rightarrow H^{1}\left(X_{1} \cap\left(X_{2} \cup \cdots \cup X_{n}\right) ; \mathcal{A}_{E}^{<0}\right)
\end{aligned}
$$

is in this case

$$
\begin{aligned}
0 \rightarrow H^{1}\left(\partial \tilde{\boldsymbol{C}}^{n} ; \mathcal{A}_{E}^{<0}\right) \stackrel{\varepsilon}{\longrightarrow} \boldsymbol{C}[[z]] / \mathcal{O}\left(\boldsymbol{C}^{n-1}\right)\left\{z_{1}\right\} \oplus \boldsymbol{C}[[z]] / \bigcap_{k=2}^{n} \mathcal{O}\left(\boldsymbol{C}^{n-1}\right)\left\{z_{k}\right\} \\
\stackrel{\alpha}{\longrightarrow} \boldsymbol{C}[[z]] / \bigcap_{k=2}^{n} \mathcal{O}\left(\boldsymbol{C}^{n-2}\right)\left\{z_{1}, z_{k}\right\} .
\end{aligned}
$$

Let

$$
\beta: C[[z]] \rightarrow \boldsymbol{C}[[z]] / \mathcal{O}\left(\boldsymbol{C}^{n-1}\right)\left\{z_{1}\right\} \oplus \boldsymbol{C}[[z]] / \bigcap_{k=2}^{n} \mathcal{O}\left(\boldsymbol{C}^{n-1}\right)\left\{z_{k}\right\}
$$

be the natural quotient map. It is clear that $\alpha \circ \beta=0$. Moreover, if

$$
f=\left(f_{1}+\mathcal{O}\left(\boldsymbol{C}^{n-1}\right)\left\{z_{1}\right\}, f_{2}+\bigcap_{k=2}^{n} \mathcal{O}\left(\boldsymbol{C}^{n-1}\right)\left\{z_{k}\right\}\right) \in \operatorname{ker} \alpha
$$

then $f_{1}-f_{2} \in \bigcap_{k=2}^{n} \mathcal{O}\left(\boldsymbol{C}^{n-2}\right)\left\{z_{1}, z_{k}\right\}$ and by Lemma $2.8, f_{1}-f_{2}=h_{1}-h_{2}$ with

$$
h_{1} \in \mathcal{O}\left(\boldsymbol{C}^{n-1}\right)\left\{z_{1}\right\} ; \quad h_{2} \in \bigcap_{k=2}^{n} \mathcal{O}\left(\boldsymbol{C}^{n-1}\right)\left\{z_{k}\right\}
$$

and $f=\beta\left(f_{1}-h_{1}=f_{2}-h_{2}\right)$. Then, $\operatorname{im} \beta=\operatorname{ker} \alpha=\operatorname{im} \varepsilon$, and there is an injective map

$$
\gamma: \boldsymbol{C}[[z]] \rightarrow H^{1}\left(\partial \tilde{\boldsymbol{C}}^{n} ; \mathcal{A}_{E}^{<0}\right)
$$

such that $\varepsilon \circ \gamma=\beta$. So, $\operatorname{ker} \gamma=\operatorname{ker} \beta=\bigcap_{k=1}^{n} \mathcal{O}\left(\boldsymbol{C}^{n-1}\right)\left\{z_{k}\right\}$ and this ends the result for $E=(0)$ (the Gevrey case is analogous).

For $E$ general, we apply a cohomological argument as in Proposition 2.4.

The only remaining thing is the proof of Proposition 2.10 . The sheaf $\mathcal{A} / \mathcal{A}_{E}^{<0}$ over $\tilde{\boldsymbol{C}}^{n}$ $(E=(0))$ has stalks

$$
\left(\mathcal{A} / \mathcal{A}_{E}^{<0}\right)_{z}=\mathcal{O}_{\{1, \ldots, n\} \backslash J}\left[\left[z_{J}\right]\right]
$$

or

$$
\left(J=\left\{j \in\{1, \ldots, n\} \mid z_{j} \in S^{1} \times\{0\} \subseteq \tilde{\boldsymbol{C}}\right\}\right) \text {. }
$$

$$
\left(\mathcal{A}_{s} / \mathcal{A}_{E}^{\leq-\kappa}\right)_{z}=\mathcal{O}_{\{1, \ldots, n\} \backslash J}\left[\left[z_{J}\right]\right]
$$

We argue as before. For a 1-cocycle $f_{(j, k)}^{\left(j^{\prime}, k^{\prime}\right)}$ of the sheaf $\mathcal{A}$, that is cohomologically trivial over $\mathcal{A} / \mathcal{A}_{E}^{<0}$, by the previous results (again with parameters) we can find, for $J \varsubsetneqq\{1, \ldots, n\}$, $f_{(j, k)_{J},\left(j^{\prime}, 0\right)_{J c}}$ such that

$$
f_{(j, k)_{J},\left(j^{\prime}, 0\right)_{J c}}-f_{(j, k)_{J},(j, 0)_{J} c}=f_{(j, k)_{J},(j, 0)_{J} c}^{(j, k)_{J},\left(j^{\prime}, 0\right)_{J^{c}}}
$$

$\left((j, k)_{J}\right.$ fixed, $k_{l} \neq 0$ if $\left.l \in J\right)$.

For each $J \subseteq\{1, \ldots, n\}, k_{l} \neq 0$ if $l \notin J$, we have open sets $D_{J} \times U_{(j, k)_{J c}}$, where $D_{J}$ is a polydisk obtained by glueing the polysectors corresponding to $\prod_{i \in J}\left(I_{j_{i}}^{i} \times A_{0}^{i}\right)$ and 
$U_{(j, k)_{J} c}$ is the open set in $C^{J^{c}}$ obtained from $\prod_{i \in J}\left(I_{j_{i}}^{l} \times A_{k_{l}}^{l}\right)$. If $\mathcal{U}$ is the open covering of $C^{n}$ consisting in the just defined sets, it is well-known that $H^{1}(\mathcal{U} ; \mathcal{O})=0$. We define a 1-cocycle on $\mathcal{U}$ with values in the sheaf $\mathcal{O}$ as follows:

i. If $J=\emptyset$, then $g_{(j, k)}^{\left(j^{\prime}, k^{\prime}\right)}:=f_{(j, k)}^{\left(j^{\prime}, k^{\prime}\right)}$.

ii. If $J \neq \emptyset$, then on $\left(D_{J_{1}} \times U_{(j, k)_{J_{1}^{c}}}\right) \cap\left(D_{J_{2}} \times U_{\left(j^{\prime}, k^{\prime}\right)_{J_{2}^{c}}}\right)$,

$$
g_{(j, k)_{J_{1}^{c}}}^{\left(j^{\prime}, k^{\prime}\right)_{J^{c}}^{c}}:=f_{(j, 0)_{J_{1}},(j, k)_{J_{1}^{c}}}^{\left(j^{\prime}, 0\right)_{J_{2}},\left(j^{\prime}, k^{\prime}\right)_{c}^{c}}-f_{\left(j^{\prime}, 0\right)_{J_{2}},\left(j^{\prime}, k^{\prime}\right)_{J_{2}^{c}}}+f_{(j, 0)_{J_{1}},(j, k)_{J_{1}^{c}}}
$$

(as before, independent of $(j, 0)_{J_{1}},\left(j^{\prime}, 0\right)_{J_{2}}$ ).

So, we have

$$
g_{(j, k)_{J_{1}^{c}}^{c}}^{\left(j^{\prime}, k^{\prime}\right)_{J^{c}}^{c}}=g_{\left(j^{\prime}, k^{\prime}\right)_{J_{2}^{c}}}-g_{(j, k)_{J_{1}^{c}}} .
$$

As before, the cochain defined by

$$
F_{(j, 0)_{J},(j, k)_{J^{c}}}:=f_{(j, 0)_{J},(j, k)_{J^{c}}}+g_{(j, k)_{J}}
$$

has as coboundary the desired cocycle.

REMARK 2.11. Again, the spaces $\Gamma\left(\partial \tilde{\boldsymbol{C}}^{n} ; \mathcal{A} / \mathcal{A}_{E}^{<0}\right)$ and $\Gamma\left(\partial \tilde{\boldsymbol{C}}^{n} ; \mathcal{A}_{s} / \mathcal{A}_{E}^{\leq-\kappa}\right)$ may be computed explicitly, and they agree with the space of sections over $\boldsymbol{T}^{n}$.

REMARK 2.12. If, instead of $\partial \tilde{\boldsymbol{C}}^{n}$, we restrict ourselves to a neighbourhood of the origin, say $D$, we obtain the same with $C^{n}$ replaced by $D$, i.e.,

$$
\begin{gathered}
H^{1}\left(\partial \tilde{D} ; \mathcal{A}_{E}^{<0}\right) \cong \Gamma\left(\partial \tilde{\boldsymbol{C}}^{n} ; \mathcal{A} / \mathcal{A}_{E}^{<0}\right) / \bigcap_{i=1}^{n} \mathcal{O}\left(D_{1} \times \cdots \times \hat{D}_{i} \times \cdots \times D_{n}\right)\left\{z_{i}\right\}, \\
H^{1}\left(\partial \tilde{D} ; \mathcal{A}_{E}^{\leq-\kappa}\right) \cong \Gamma\left(\partial \tilde{\boldsymbol{C}}^{n} ; \mathcal{A}_{s} / \mathcal{A}_{E}^{\leq-\kappa}\right) / \bigcap_{i=1}^{n} \mathcal{O}\left(D_{1} \times \cdots \times \hat{D}_{i} \times \cdots \times D_{n}\right)\left\{z_{i}\right\} .
\end{gathered}
$$

If we take the inductive limit $\left(\partial \tilde{C}^{n}, 0\right)=\underset{r}{\lim } \partial \tilde{D}_{r}$, we obtain

$$
\begin{gathered}
H^{1}\left(\left(\partial \tilde{\boldsymbol{C}}^{n}, 0\right) ; \mathcal{A}_{E}^{<0}\right) \cong \lim _{\longrightarrow} H^{1}\left(\partial \tilde{D}_{r} ; \mathcal{A}_{E}^{<0}\right)=\Gamma\left(\partial \tilde{\boldsymbol{C}}^{n} ; \mathcal{A} / \mathcal{A}_{E}^{<0}\right) / \boldsymbol{C}\{z\}, \\
H^{1}\left(\left(\partial \tilde{\boldsymbol{C}}^{n}, 0\right) ; \mathcal{A}_{E}^{\leq-\kappa}\right) \cong \lim _{\longrightarrow} H^{1}\left(\partial \tilde{D}_{r} ; \mathcal{A}_{E}^{\leq-\kappa}\right)=\Gamma\left(\partial \tilde{\boldsymbol{C}}^{n} ; \mathcal{A}_{s} / \mathcal{A}_{E}^{\leq-\kappa}\right) / \boldsymbol{C}\{z\},
\end{gathered}
$$

as $H^{1}$ commutes with $\underset{\lim }{\longrightarrow}$

3. First cohomology set in the non-abelian case. Now, we shall consider sheaves of invertible matrices whose coefficients have asymptotic development. These are sheaves of non-abelian groups, for which only the 0th and the 1st order Čech cohomology are well defined (several non equivalent definitions of $H^{2}$ have been given). Moreover, $H^{1}$ is not a group, but a pointed set (set with a distinguished element). The main definitions and results of non-abelian sheaf cohomology can be seen in [F, Gi, Hz].

The sheaves we shall mainly use are: 
i. $G L(m, \mathcal{A}), G L\left(m, \mathcal{A}_{s}\right)$ of invertible $m \times m$ matrices with coefficients in $\mathcal{A}, \mathcal{A}_{s}$, respectively.

ii. $G L(m, \mathcal{A})_{I_{m}, E}, G L\left(m, \mathcal{A}_{s}\right)_{I_{m}, E}$ of invertible matrices that have the identity matrix $I_{m}$ as asymptotic development in the divisor or subvariety $E$. These are the invertible matrices $M$ such that $M-I_{m}$ have coefficients in $\mathcal{A}_{E}^{<0}, \mathcal{A}_{E}^{\leq-\kappa}$, respectively.

3.1. Main non-abelian lemma. The main lemma of Subsection 2.1 may be rephrased as follows.

LEMMA 3.1. Let $f \in G L(m, \mathcal{A})_{I_{m},\left(z_{1}=0\right)}(V)$ and $V_{11}, V_{12}$ be sectors in $C$ with $V_{11} \cap$ $V_{12}=V_{1}$. If $W^{\prime}<V, W_{11}^{\prime}<V_{11}, W_{12}^{\prime}<V_{12}$ and $W_{11}^{\prime} \cap W_{12}^{\prime}=W_{1}^{\prime}$, then there exist $f_{i}(z) \in G L(m, \mathcal{A})_{I_{m},\left(z_{1}=0\right)}(W)$ (perhaps reducing the radius of $W$ ) such that $f=f_{1}^{-1} \cdot f_{2}$.

Proof. We follow here the idea of the proof of Sibuya $[S]$ for the case of one variable. We will omit the details that are similar to those encountered there.

Take a sequence of subsectors $W_{1}>W_{2}>\cdots$ such that $\bigcap_{k=1}^{\infty} W_{k}=W^{\prime}$. If $g_{1}(z)=$ $f(z)-I_{m}$, write (by Lemma 2.1) $g_{1}(z)=g_{12}(z)-g_{11}(z)$ on $W_{1} \times V_{2} \times \cdots \times V_{n}$. Define $g_{2}(z)=g_{11}(z) \cdot g_{1}(z) \cdot\left(I_{m}+g_{12}(z)\right)^{-1}$ and write $g_{2}(z)=g_{22}(z)-g_{21}(z)$ on $W_{2} \times V_{2} \times \cdots \times V_{n}$.

Iterating this process, you have a sequence $\left\{g_{k}(z)=g_{k 2}(z)-g_{k 1}(z)\right\}_{k=1}^{\infty}$. Define now

$$
f_{1}(z)=\lim _{k \rightarrow \infty}\left(I_{m}+g_{k i}(z)\right) \cdot\left(I_{m}+g_{k-1, i}(z)\right) \cdots\left(I_{m}+g_{k 1}(z)\right) .
$$

By the equality

$$
\left(I_{m}+g_{k+1}(z)\right) \cdot\left(I_{m}+g_{k 2}(z)\right)=\left(I_{m}+g_{k 1}(z)\right) \cdot\left(I_{m}+g_{k}(z)\right),
$$

it can be seen that $f_{1}(z) \cdot f(z)=f_{2}(z)$. Choosing $\left\{W_{k}\right\}_{k}$ carefully, the convergence of the expression defining $f_{i}(z)$ is assured, from the expression of the Cauchy-Heine transform and reducing the radius conveniently.

In order to show taht $f_{i}(z)$ has an asymptotic development, we use [M1, prop. 3] (for a proof, see [Mo]). A bound $C_{W^{\prime \prime}, N}^{k}$ for the derivative $D^{N} g_{k i}(z)$ on $W^{\prime \prime}<W_{i_{1}}^{\prime} \times W_{2}^{\prime} \times \cdots \times W_{n}^{\prime}$ can be obtained by a universal polynomial $P_{N}$ with natural coefficients

$$
C_{W^{\prime \prime}, N}^{k}=P_{N}\left(\left\{C_{W^{\prime \prime}, N^{\prime}}^{k-1} / N^{\prime} \leq N\right\}, R^{\prime \prime}\right)
$$

( $R^{\prime \prime}$ the radius), such that $R^{\prime \prime}$ divides $P_{N}$, and so, reducing $R^{\prime \prime}$, the series

$$
\sum_{k=1}^{\infty} C_{W^{\prime \prime}, N}^{k}
$$

converges, which completes the proof.

4. Computation of the cohomology over $\boldsymbol{T}^{n}$ in the non-abelian case. The multiplicative analogue of Proposition 2.2 can be stated as

Proposition 4.1. Let $\mathcal{V}=\left\{V_{1}, \ldots, V_{r}\right\}$ be a good covering of $S^{1}$, and $V^{\prime}$ a polysector in $C^{n-1}$. If $F_{i, i+1}$ are $m \times m$ matrices of functions with asymptotic development on $V_{i, i+1} \times V^{\prime}$, and

$$
F A_{1}\left(F_{12}\right) \cdot F A_{1}\left(F_{23}\right) \cdots F A_{1}\left(F_{r 1}\right)=I_{m}
$$


then there are $F_{i}$ on $V_{i} \times V^{\prime}$ such that $F_{i} \cdot F_{i, i+1}=F_{i+1}$ on $V_{i, i+1} \times V^{\prime}$.

PROOF. As in the function case, if $G_{i}$ on $V_{i} \times V^{\prime}$ is such that

$$
F A_{1}\left(G_{i}\right)=F A_{1}\left(F_{12}\right) \cdot F A_{1}\left(F_{23}\right) \cdots F A_{1}\left(F_{i-1, i}\right)
$$

and $f_{i, i+1}=G_{i} \cdot F_{i, i+1} \cdot G_{i+1}^{-1}\left(G_{1}=I_{m}\right)$, then we can suppose $F A_{1}\left(F_{i, i+1}\right)=I_{m}$. As in that case, consider first the situation where only perhaps $F_{12} \neq I_{m}$. Take $f_{1}^{\prime} \in$ $G L(m, \mathcal{A})_{I_{m},\left(z_{1}=0\right)}\left(V_{1} \times V^{\prime}\right), f_{2}^{\prime} \in G L(m, \mathcal{A})_{I_{m},\left(z_{1}=0\right)}\left(\left(V_{2} \cup \cdots \cup V_{r}\right) \times V^{\prime}\right)($ Lemma 3.1). If, in the Riemann surface of $\log z$, we write $f_{2}^{\prime}=f_{r}^{-1} \cdot f_{r}^{\prime}$, with $f_{r}$ on $V_{2} \cup \cdots \cup V_{r} \cup V_{1}$, $f_{r}^{\prime}$ on $V_{1} \cup \cdots \cup V_{r}$, the equality $f_{r} \cdot f_{2}^{\prime}=f_{r}^{\prime}$ allows us to define a function $f$ on a big sector, and its restrictions to $V_{1}$ are the functions $F_{i}$.

In the general case, we argue as in the abelian case, by "multiplicative linearity". This argument was also used by Sibuya [S] and Majima [M1].

THEOREM 4.2. In the category of pointed sets, there are isomorphisms

$$
H^{1}\left(\boldsymbol{T}^{n} ; G L(m, \mathcal{A})_{I_{m}, E} \cong \Gamma\left(\boldsymbol{T}^{n} ; G L(m, \mathcal{A}) / G L(m, \mathcal{A})_{I_{m}, E}\right) / G L(m, C\{z\}) .\right.
$$

PROOF. We shall suppose $E=(0)$. In this case, we have the equivalence

$$
\Gamma\left(\boldsymbol{T}^{n} ; G L(m, \mathcal{A}) / \mathcal{A}_{E}^{<0}\right) \cong G L(m, C[[z]]) .
$$

By a theorem of Borel-Ritt, the natural map (fixing a good covering $\mathcal{V}$ of $\boldsymbol{T}^{n}$ )

$$
G L(m, C[[z]]) \stackrel{\alpha}{\longrightarrow} H^{1}\left(T^{n} ; G L(m, \mathcal{A})_{I_{m}, E}\right)
$$

can be constructed in such a way that $\alpha(F)=\alpha(F G)$ if $G \in G L(m, C\{z\})$. So, it defines a map (again denoted by $\alpha$ )

$$
G L(m, C[[z]]) / G L(m, C\{z\}) \stackrel{\alpha}{\longrightarrow} H^{1}\left(T^{n} ; G L(m, \mathcal{A})_{I_{m}, E}\right) .
$$

It is easily checked that $\alpha$ is injective. Let us prove its surjectivity. If $\left[\left(F_{i, i^{\prime}}\right)_{i, i^{\prime}}\right] \in$

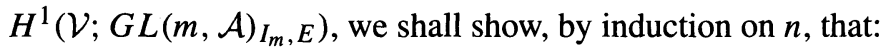

1. There are $F_{i} \in \Gamma\left(V_{i} ; G L(m, \mathcal{A})\right)$ such that $F_{i i^{\prime}}=F_{i}^{-1} \cdot F_{i^{\prime}}$.

2. Fixing $\tilde{\imath} \in N^{n-1}$, then

$$
F A_{1}\left(F_{(1, \tilde{\imath}),(2, \tilde{\imath})}\right) \cdot F A_{1}\left(F_{(2, \tilde{\imath}),(3, \tilde{\imath})}\right) \cdots F A_{1}\left(F_{(r, \tilde{l}),(1, \tilde{l})}\right)=I_{m}
$$

as a matrix of elements of $\mathcal{A}\left(V_{\tilde{i}}\right)\left[\left[z_{1}\right]\right]$.

If $n=1$, the result is trivial. For bigger $n$, let

$$
A_{\tilde{\imath}}=F A_{1}\left(F_{(1, \tilde{\imath}),(2, \tilde{\imath})}\right) \cdot F A_{1}\left(F_{(2, \tilde{i}),(3, \tilde{\imath})}\right) \cdots F A_{1}\left(F_{(r, \tilde{l})(1, \tilde{\imath})}\right) \in \mathcal{A}\left(V_{\tilde{\imath}}\right)\left[\left[z_{1}\right]\right] .
$$

From the equality

$$
F_{(k, \tilde{\imath}),\left(k, \tilde{i}^{\prime}\right)} \cdot F_{\left(k, \tilde{i}^{\prime}\right),\left(k+1, \tilde{i}^{\prime}\right)}=F_{(k, \tilde{\imath}),(k+1, \tilde{\imath})} \cdot F_{(k+1, \tilde{\imath}),\left(k+1, \tilde{i}^{\prime}\right)},
$$

we obtain

$$
A_{\tilde{\imath}} \cdot F A_{1}\left(F_{(1, \tilde{\imath}),\left(1, \tilde{\imath}^{\prime}\right)}\right)=F A_{1}\left(F_{(1, \tilde{\imath}),\left(1, \tilde{i}^{\prime}\right)}\right) \cdot A_{\tilde{\imath}^{\prime}}
$$

The family

$$
\left(F A_{1}\left(F_{(1, \tilde{i}),\left(1, \tilde{\imath}^{\prime}\right)}\right)\right) \tilde{i}, \tilde{\imath}^{\prime}
$$


is a cocycle of the sheaf $G L(m, C[[z]])$. If $L$ is a Fréchet space, $L[[z]]$ has also a natural Fréchet structure, and the space $\mathcal{A}(V)[[z]]$ can be identified with $\mathcal{A}(V ; C[[z]])$. Using the induction hypothesis (for asymptotic developments with coefficients in a Fréchet space), we obtain $F_{\tilde{\imath}} \in G L(m, \mathcal{A}(*, C[[z]]))\left(V_{\tilde{\imath}}\right)$ such that

$$
F A_{1}\left(F_{(1, \tilde{\imath}),\left(1, \tilde{\imath}^{\prime}\right)}\right)=F_{\tilde{\imath}}^{-1} \cdot F_{\tilde{\imath}^{\prime}},
$$

and so the equality

$$
F_{\tilde{\imath}} \cdot A_{\tilde{\imath}} \cdot F_{\tilde{\imath}}^{-1}=F_{\tilde{\imath}^{\prime}} \cdot A_{\tilde{l}^{\prime}} \cdot F_{\tilde{\imath}^{\prime}}^{-1}
$$

defines a matrix with elements in $C\left\{z_{2}, \ldots, z_{n}\right\}\left[\left[z_{1}\right]\right]$. As $A_{\tilde{\imath}}$ has the identity matrix as an asymptotic development at the origin, $F_{\tilde{\imath}} \cdot A_{\tilde{\imath}} F_{\tilde{\imath}}^{-1}=I_{m}$ and hence $A_{\tilde{\imath}}=I_{m}$.

The rest of the reasoning is exactly as in the abelian case. We obtain $F_{(k, \tilde{\imath})}$ with

$$
F_{(k, \tilde{\imath})} \cdot F_{(k, \tilde{\imath})(k+1, \tilde{\imath})}
$$

and the identity

$$
F_{\left(i_{1}, \tilde{\imath}\right)} \cdot F_{\left(i_{1}, \tilde{i}\right),\left(i_{1}^{\prime}, \tilde{\imath}^{\prime}\right)} \cdot F_{\left(i_{1}^{\prime}, \tilde{\imath}^{\prime}\right)}^{-1}=F_{\left(i_{1}^{\prime \prime}, \tilde{i}\right)} \cdot F_{\left(i_{1}^{\prime \prime}, \tilde{i}\right),\left(i_{1}^{\prime \prime \prime}, \tilde{i}^{\prime}\right)} \cdot F_{\left(i_{1}^{\prime \prime \prime}, \tilde{\imath}^{\prime}\right)}^{-1}
$$

allows us to reason by recurrence. At last, we construct $F_{i} \in G L(m, \mathcal{A})\left(V_{i}\right)$ with $F_{i, i^{\prime}}=$ $F_{i}^{-1} F_{i^{\prime}}$. If $\hat{F} \in C[[z]]$ is the series of asymptotic development at the origin of $F_{i}, \alpha(\hat{F})=$ $\left(F_{i, i^{\prime}}\right)_{i, i^{\prime}}$ and so $\alpha$ is surjective.

For $E$ general, it is possible to use a cohomological argument as in the function case (there is a "long" exact sequence in the category of pointed sets, see $[F]$ ), or to repeat the preceding argument for each $E$.

REMARK 4.3. As in the abelian case, if $E=H_{1} \cup \cdots \cup H_{n}$, the result can be read in [M1] and [Ha] (general and Gevrey cases, respectively).

5. General and Gevrey cases. The only essential difference with the function case is in the use of Mayer-Vietoris principle. Such a result, for the non-abelian sheaf cohomology, seems to be known by the specialists, but we have not found a precise reference. With this result in hand, we could show:

THEOREM 5.1. There is an isomorphism of pointed sets

$$
H^{1}\left(\partial \tilde{\boldsymbol{C}}^{n} ; G L(m, \mathcal{A})_{I_{m}, E}\right) \cong \Gamma\left(\partial \tilde{\boldsymbol{C}}^{n} ; G L(m, \mathcal{A}) / \mathcal{A}_{E}^{<0}\right) / G L\left(m, \bigcap_{i=1}^{n} \mathcal{O}\left(\boldsymbol{C}^{n-1}\right)\left\{z_{i}\right\}\right) \text {. }
$$

The Gevrey case is deduced from the general asymptotic case by the following observation: If $\left(f_{i, i^{\prime}}\right)_{i, i^{\prime}}$ is a Gevrey 1-cocycle, we can write $\left(f_{i, i^{\prime}}\right)_{i, i^{\prime}}=f_{i}^{-1} \cdot f_{i^{\prime}}$ where $f_{i}$ has asymptotic expansion (not necessarily Gevrey). If $\left(f_{i, i^{\prime}}\right)_{i, i^{\prime}}=I_{m}+g_{i, i^{\prime}}$, we have $f_{i} \cdot g_{i, i^{\prime}}=f_{i^{\prime}}-f_{i}$ with null asymptotic development $s$-Gevrey over the corresponding divisor or subvariety $E$. So, $f_{i^{\prime}}-f_{i}=g_{i^{\prime}}-g_{i}, g_{i} s$-Gevrey (by the function case). Then, $f_{i}=u+g_{i}$, with $u$ convergent ( $u$ is formed by glueing $f_{i}-g_{i}=f_{i^{\prime}}-g_{i^{\prime}}$ ) and $g_{i}$ is $s$-Gevrey, so $f_{i}$ is $s$-Gevrey.

THEOREM 5.2. There are isomorphisms of pointed sets

$$
H^{1}\left(\boldsymbol{T}^{n} ; G L\left(m, \mathcal{A}_{s}\right)_{I_{m}, E}\right) \cong \Gamma\left(\boldsymbol{T}^{n} ; G L\left(m, \mathcal{A}_{s}\right) / G L\left(m, \mathcal{A}_{s}\right)_{I_{m}, E}\right) / G L(m, C\{z\}),
$$




$$
\begin{aligned}
& H^{1}\left(\partial \tilde{\boldsymbol{C}}^{n} ; G L\left(m, \mathcal{A}_{s}\right)_{I_{m}, E}\right) \\
& \quad \cong \Gamma\left(\boldsymbol{T}^{n} ; G L\left(m, \mathcal{A}_{s}\right) / G L\left(m, \mathcal{A}_{s}\right)_{I_{m}, E}\right) / G L\left(m, \bigcap_{i=1}^{n} \mathcal{O}\left(\boldsymbol{C}^{n-1}\right)\left\{z_{i}\right\}\right) .
\end{aligned}
$$

\section{REFERENCES}

[F] J. FrEnKEL, Cohomologie non abélienne et espaces fibrés, Bull. Soc. Math. France 85 (1957), 135-218.

[Gi] J. GiRAUd, Cohomologie non abélienne, Grundlehren Math. Wiss. 179, Springer-Verlag, Berlin-New York, 1971.

[Gr] A. GRothendieck, Éléments de Géométrie Algébrique I, Springer-Verlag, 1971.

[Ha] Y. HARAOKA, Theorems of Sibuya-Malgrange type for Gevrey functions of several variables, Funkcial. Ekvac. 32 (1989), 365-388.

[He] J. HeRnándeZ Isla, Desarrollos asintóticos en polisectores, Problemas de existencia y unicidad, Tesis de doctorado, Universidad de Valladolid, 1994.

[Hz] F. HiRzEBRUCH, Topological methods in algebraic geometry, Grundlehren Math. Wiss. 131, SpringerVerlag, 1966.

[I] B. IVERSEN, Cohomology of sheaves, Springer-Verlag Universitext, 1986.

[M] H. Majima, Remarques sur la théorie de Développement asymptotique en plusieurs variables I, Proc. Japan Acad. Ser. A Math. Sci. 54 (1978), 67-72.

[M1] H. MaJimA, Analogues of Cartan's decomposition theorem in asymptotic analysis, Funkcial. Ekvac. 26 (1983), 131-154.

[M2] H. MAJIMA, Asymptotic Analysis for Integrable Connections with Irregular Singular Points, Lecture Notes in Math. 1075, Springer-Verlag, 1984.

[MI] B. MALGRANGE, Remarques sur les équations différentielles à points singuliers irréguliers, in Équations différentielles et systèmes de Pfaff dans le champ complexe (R. Gérard and J. P. Ramis, editors), Lecture Notes in Math. 712, Springer-Verlag, 1979.

[Mo] J. MOZo FERNÁNDEZ, Teoremas de división y de Malgrange-Sibuya para funciones con desarrollo asintótico fuerte en varias variables, Tesis de doctorado, Universidad de Valladolid, 1996.

[S] Y. SibUYA, Linear differential equations in the complex domain: Problems of analytic continuation, Progress in Mathematics, Transl. Math. Monogr. 82, Amer. Math. Soc., Providence, RI, 1990.

[Z] M. A. ZURro Moro, Series y funciones Gevrey en varias variables, Tesis de doctorado, Universidad de Valladolid, 1994.

Departamento de Matemática Aplicada Fundamental

ETS DE ARQUITECTURA

AVEnida Salamanca S/N

47014 VALLADOLID

SPAIN

E-mail address: jmozo@maf.uva.es 